Open Access

Helong Bai and Ping Zhang*

\title{
Crystal structure of poly-[bis $\left\{\mu_{2}-\mathrm{N}-(4-(1 H-\right.$ imidazol-1-yl)phenyl)-4-(1H-imidazol-1-yl)-N- phenylaniline- $\left.\left.\mathrm{K}^{2} N: N^{\prime}\right)\right\}-\left(\mu_{2}\right.$-naphthalene-2,6- dicarboxylato)-( $\mu_{4}$-naphthalene-2,6- dicarboxylato)dicadmium(II)], $\mathrm{C}_{36} \mathrm{H}_{25} \mathrm{~N}_{5} \mathrm{O}_{4} \mathrm{Cd}$
}

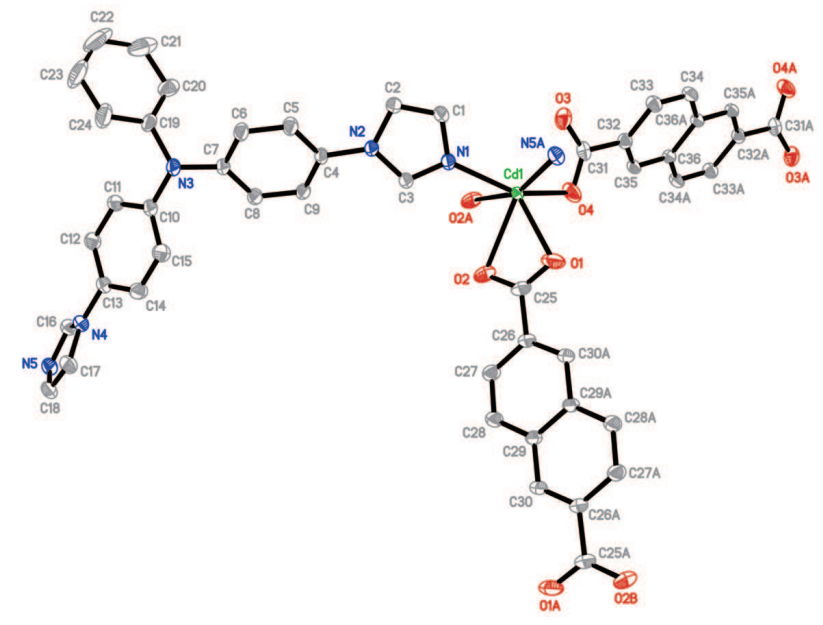

DOI 10.1515/ncrs-2016-0034

Received January 29, 2016; accepted May 31, 2016; available online June 14, 2016

\section{Abstract}

$\mathrm{C}_{36} \mathrm{H}_{25} \mathrm{~N}_{5} \mathrm{O}_{4} \mathrm{Cd}$, monoclinic, $P \overline{1}$ (No. 2), $a=9.708(5) \AA$, $b=10.954(5) \AA, c=15.014(5) \AA, \alpha=97.858(5)^{\circ}, \beta=98.584(5)^{\circ}$, $\gamma=104.798(5)^{\circ}, V=1500.6(11) \AA^{3}, \quad Z=2, \quad R_{\mathrm{gt}}(F)=0.0297$, $w R_{\text {ref }}\left(F^{2}\right)=0.0904, T=293(2) \mathrm{K}$.

\section{CCDC no.: 1482446}

A part of the crystal structure is shown in the figure. Tables 1 and 2 contain details of the measurement method and a list of the atoms including atomic coordinates and displacement parameters.

\footnotetext{
*Corresponding author: Ping Zhang, College of Chemistry,
} Jilin University, Changchun 130012, China; and Department of Chemistry, Changchun Normal University, Changchun 130032, China, e-mail: drzhangp@sina.com

Helong Bai: College of Chemistry, Jilin University, Changchun 130012, China; and Department of Chemistry, Changchun Normal University, Changchun 130032, China
Table 1: Data collection and handling.
Crystal:

Wavelength:

$\mu$ :

Diffractometer, scan mode:

$2 \theta_{\max }$, completeness:

$N(h k l)_{\text {measured }}, N(h k l)_{\text {unique }}, R_{\text {int }}$ :

Criterion for $l_{\text {obs }}, N(h k l)_{\mathrm{gt}}$ :

$N(\text { param })_{\text {refined: }}$

Programs:
Colorless blocks Size $0.28 \times 0.22 \times 0.21 \mathrm{~mm}$ Mo $K_{\alpha}$ radiation $(0.71073 \AA)$ $7.8 \mathrm{~cm}^{-1}$ Bruker CCD, $\varphi$ and $\omega$ $56.6^{\circ},>99 \%$ $11248,7455,0.015$

$I_{\text {obs }}>2 \sigma\left(I_{\text {obs }}\right), 6339$ 416 SHELX [11]
Table 2: Fractional atomic coordinates and isotropic or equivalent isotropic displacement parameters $\left(\AA^{2}\right)$.

\begin{tabular}{lrrrr}
\hline Atom & $\boldsymbol{x}$ & $\boldsymbol{y}$ & $\boldsymbol{z}$ & \multicolumn{1}{c}{$\boldsymbol{U}_{\text {iso }}{ }^{*} \boldsymbol{U}_{\text {eq }}$} \\
\hline C1 & $0.0799(4)$ & $0.1418(3)$ & $-0.04865(19)$ & $0.0551(8)$ \\
H1 & 0.1130 & 0.1115 & 0.0030 & $0.066^{*}$ \\
C2 & $0.0235(4)$ & $0.0687(3)$ & $-0.13296(19)$ & $0.0521(7)$ \\
H2 & 0.0106 & -0.0189 & -0.1498 & $0.063^{*}$ \\
C3 & $0.0253(3)$ & $0.2685(2)$ & $-0.13553(16)$ & $0.0371(5)$ \\
H3 & 0.0122 & 0.3416 & -0.1566 & $0.044^{*}$ \\
C4 & $-0.0751(3)$ & $0.1157(2)$ & $-0.28399(16)$ & $0.0339(5)$ \\
C5 & $-0.0546(3)$ & $0.0095(3)$ & $-0.33598(18)$ & $0.0428(6)$ \\
H5 & 0.0045 & -0.0359 & -0.3096 & $0.051^{*}$ \\
C6 & $-0.1217(3)$ & $-0.0288(3)$ & $-0.42687(17)$ & $0.0426(6)$ \\
H6 & -0.1057 & -0.0991 & -0.4617 & $0.051^{*}$ \\
C7 & $-0.2132(3)$ & $0.0362(2)$ & $-0.46752(16)$ & $0.0364(5)$ \\
C8 & $-0.2271(3)$ & $0.1458(2)$ & $-0.41577(17)$ & $0.0384(5)$ \\
H8 & -0.2826 & 0.1935 & -0.4428 & $0.046^{*}$ \\
C9 & $-0.1597(3)$ & $0.1856(2)$ & $-0.32440(17)$ & $0.0362(5)$ \\
H9 & -0.1711 & 0.2587 & -0.2904 & $0.043^{*}$ \\
C10 & $-0.3456(3)$ & $0.0594(2)$ & $-0.61679(17)$ & $0.0373(5)$ \\
C11 & $-0.4800(3)$ & $0.0095(3)$ & $-0.67544(18)$ & $0.0414(6)$ \\
H11 & -0.5358 & -0.0730 & -0.6746 & $0.050^{*}$ \\
C12 & $-0.5320(3)$ & $0.0805(3)$ & $-0.73488(18)$ & $0.0422(6)$ \\
H12 & -0.6213 & 0.0453 & -0.7743 & $0.051^{*}$ \\
C13 & $-0.4516(3)$ & $0.2032(2)$ & $-0.73568(17)$ & $0.0375(5)$
\end{tabular}


Table 2: (continued)

\begin{tabular}{|c|c|c|c|c|}
\hline Atom & $x$ & $y$ & $z$ & $\boldsymbol{U}_{\text {iso }} * / \boldsymbol{U}_{\mathrm{eq}}$ \\
\hline 14 & $-0.3189(3)$ & $0.2542(3)$ & $-0.6772(2)$ & $0.0499(7)$ \\
\hline H14 & -0.2650 & 0.3378 & -0.6768 & $0.060^{*}$ \\
\hline C15 & $-0.2652(3)$ & $0.1824(3)$ & $-0.6192(2)$ & $0.0490(7)$ \\
\hline 15 & -0.1742 & 0.2171 & -0.5816 & $0.059^{*}$ \\
\hline C16 & $-0.6427(3)$ & $0.2793(3)$ & $-0.81927(19)$ & $0.0423(6)$ \\
\hline 16 & -0.7197 & 0.2295 & -0.7982 & $0.051^{*}$ \\
\hline 17 & $-0.4229(3)$ & $0.3644(3)$ & $-0.84062(19)$ & $0.0450(6)$ \\
\hline 17 & -0.3231 & 0.3849 & -0.8380 & $0.054^{*}$ \\
\hline 19 & $-0.3521(3)$ & $-0.1526(3)$ & $-0.58199(19)$ & $0.0487(7)$ \\
\hline 20 & $-0.4267(3)$ & $-0.2204(3)$ & $-0.5248(3)$ & $0.0589(8)$ \\
\hline 20 & -0.4321 & -0.1794 & -0.4675 & $0.071^{*}$ \\
\hline $\mathrm{C} 21$ & $-0.4940(5)$ & $-0.3515(3)$ & $-0.5541(4)$ & $0.0926(15)$ \\
\hline 21 & -0.5453 & -0.3981 & -0.5162 & $0.111^{*}$ \\
\hline $\mathrm{C} 22$ & $-0.4852(7)$ & $-0.4119(4)$ & $-0.6377(5)$ & $0.135(3)$ \\
\hline 22 & -0.5311 & -0.4992 & -0.6569 & $0.162^{*}$ \\
\hline $\mathrm{C} 23$ & $-0.4090(8)$ & $-0.3444(5)$ & $-0.6932(4)$ & $0.134(3)$ \\
\hline 23 & -0.4038 & -0.3862 & -0.7503 & $0.161^{*}$ \\
\hline C24 & $-0.3394(5)$ & $-0.2145(4)$ & $-0.6656(3)$ & $0.0845(13)$ \\
\hline $\mathrm{H} 24$ & -0.2849 & -0.1696 & -0.7028 & $0.101^{*}$ \\
\hline 25 & 0.246 & $0.6620(2)$ & $0.0281(2)$ & $0.0409(6)$ \\
\hline $\mathrm{C} 26$ & 0.320 & 0.79 & 0.008 & $53(5)$ \\
\hline $\mathrm{C} 27$ & $0.2563(3)$ & $0.8399(2)$ & $-0.06475(19)$ & $0.0423(6)$ \\
\hline $\mathrm{H} 27$ & 0.1643 & 0.7948 & -0.0979 & $.051 *$ \\
\hline $\mathrm{C} 28$ & $0.3287(3)$ & $0.9513(2)$ & $-0.08747(19)$ & $0.0428(6)$ \\
\hline $\mathrm{H} 28$ & 0.2860 & 0.9808 & -0.1367 & $0.051^{*}$ \\
\hline C29 & $0.4684(3)$ & $1.0234(2)$ & $-0.03752(17)$ & $332(5)$ \\
\hline C30 & $0.5460(3)$ & $1.1405(2)$ & $-0.05870(18)$ & $0.0378(5)$ \\
\hline H3O & 0.5051 & 1.1721 & -0.1074 & $0.045^{*}$ \\
\hline C31 & $0.0700(3)$ & $0.4015(3)$ & 0.247 & $08(6)$ \\
\hline 32 & $0.0587(3)$ & $0.4275(2)$ & $0.34662(16)$ & $0.0347(5)$ \\
\hline C33 & $0.1196(3)$ & $0.3609(3)$ & $0.40927(17)$ & $0.0394(6)$ \\
\hline H33 & 0.1683 & 0.3028 & 0.3889 & $0.047^{*}$ \\
\hline C34 & $0.1075(3)$ & $0.3809(2)$ & $0.49900(17)$ & $0.0381(5)$ \\
\hline H34 & 0.1504 & 0.3383 & 0.5396 & $0.046^{\star}$ \\
\hline C35 & $-0.0110(3)$ & $0.5140(2)$ & $0.37699(16)$ & $0.0333(5)$ \\
\hline H35 & -0.0466 & 0.5608 & 0.3364 & 0.040 * \\
\hline C36 & $-0.0304(2)$ & $0.5341(2)$ & $0.46909(16)$ & $0.0311(5)$ \\
\hline C18 & $-0.5183(3)$ & $0.4134(3)$ & $-0.88838(19)$ & $0.0480(7)$ \\
\hline H18 & -0.4937 & 0.4745 & -0.9250 & $0.058^{\star}$ \\
\hline N1 & $0.0813(2)$ & $0.2671(2)$ & $-0.05055(14)$ & $0.0402(5)$ \\
\hline N2 & $-0.0109(2)$ & $0.15086(19)$ & $-0.18854(13)$ & $0.0357(4)$ \\
\hline N3 & $-0.2916(3)$ & $-0.0159(2)$ & $-0.55801(15)$ & $0.0443(5)$ \\
\hline N4 & $-0.5037(2)$ & $0.2778(2)$ & $-0.79655(14)$ & $0.0387(5)$ \\
\hline N5 & $-0.6557(3)$ & $0.3610(2)$ & $-0.87553(16)$ & $0.0452(5)$ \\
\hline 01 & $0.3063(3)$ & $0.6245(2)$ & 0.09341(19) & $0.0816(9)$ \\
\hline 02 & $0.1322(2)$ & $0.59283(18)$ & $-0.02259(16)$ & $0.0529(5)$ \\
\hline 03 & $0.0705(2)$ & $0.2928(2)$ & $0.21219(13)$ & $0.0524(5)$ \\
\hline 04 & $0.0782(3)$ & $0.4951(2)$ & $0.20553(13)$ & $0.0572(6)$ \\
\hline Cd1 & $0.135574(19)$ & $0.425080(15)$ & $0.073588(11)$ & $0.03183(7)$ \\
\hline
\end{tabular}

\section{Source of material}

A mixture of naphthalene-2,6-dicarboxylic acid $(0.2 \mathrm{mmol}$, $0.043 \mathrm{~g}), \quad N$-(4-(1H-imidazol-1-yl)phenyl)-4-(1H-imidazol-1yl)- $N$-phenylaniline $(0.2 \mathrm{mmol}, 0.076 \mathrm{~g}), \mathrm{Cd}\left(\mathrm{CH}_{3} \mathrm{COO}\right)_{2}$.
$\left(\mathrm{H}_{2} \mathrm{O}\right),(0.2 \mathrm{mmol}, 0.053 \mathrm{~g})$, and $\mathrm{H}_{2} \mathrm{O}(20 \mathrm{~mL})$ was stirred for $20 \mathrm{~min}$. The mixtures were transfered in a $30 \mathrm{~mL}$ stainless steel reactor with a Teflon liner and heated from 293 to $463 \mathrm{~K}$ in $4 \mathrm{~h}$ and a constant temperature was maintained for $72 \mathrm{~h}$. After cooling to room temperature colorless block crystals were collected in $56.8 \%$ yield based on $\mathrm{Cd}\left(\mathrm{CH}_{3} \mathrm{COO}\right)_{2} \cdot 2\left(\mathrm{H}_{2} \mathrm{O}\right)$.

\section{Experimental details}

The C-bound $\mathrm{H}$ atoms were positioned with idealized geometry and were refined with $U_{\text {iso }}(\mathrm{H})=1.2 U_{\text {eq }}(\mathrm{C})$ using a riding model with $\mathrm{C}-\mathrm{H}=0.93$ Å for aromatic $\mathrm{H}$ atoms.

\section{Discussion}

Recently, many efforts have been devoted to design and synthesize coordination polymers, not only due to their potential applications in the realm of gas adsorption and separation, catalysis, magnetism, luminescence, host-guest chemistry etc, but also for their aesthetic and often complicated architectures and topologies [1-4]. It is well known that carboxylates are excellent building blocks for the construction of coordination polymers because the carboxylate groups may induce core aggregation and link these discrete clusters into an extended framework by virtue of its bridging ability [5-10]. Taking these into account, we chose naphthalene2,6-dicarboxylate and N-(4-(1H-imidazol-1-yl)phenyl)-4-(1Himidazol-1-yl)-N-phenylaniline as neutral ligand, generating the title compound.

In the title compound, Cd(II) displays a slightly distorted octahedral geometry defined by two $\mathrm{N}$ atoms from two $N$-(4-(1H-imidazol-1-yl)phenyl)-4-(1H-imidazol-1yl)- $\mathrm{N}$-phenylaniline ligands and four $\mathrm{O}$ atoms from three naphthalene-2,6-dicarboxylato ligands. The bond lengths and angles are in a normal range. In the crystal, the $\mathrm{Cd}(\mathrm{II})$ ions are bridged by naphthalene-2,6-dicarboxylate and neutral ligands forming a three-dimensional supramolecular network.

Acknowledgements: This work is supported by College of Chemistry, Jilin University.

\section{References}

1. Chen, M. M.; Chen, S. S.; Sheng, L. Q.: Crystal structure of catena-poly[aqua- $\mu-1,4$-benzenedicarboxylato- $\kappa 20: 0^{\prime}$ 3,5-dimethyl-4-phenyl -1,2,4-triazole-kN-zinc(II)] monohydrate, $\mathrm{Zn}\left(\mathrm{H}_{2} \mathrm{O}\right)\left(\mathrm{C}_{8} \mathrm{H}_{4} \mathrm{O}_{4}\right)\left(\mathrm{C}_{10} \mathrm{H}_{11} \mathrm{~N}_{3}\right) \cdot \mathrm{H}_{2} \mathrm{O}, \mathrm{C}_{18} \mathrm{H}_{17} \mathrm{~N}_{3} \mathrm{O}_{6} \mathrm{Zn}$. Z. Kristallogr. NCS 228 (2013) 337-338.

2. Song, X. Z.; Song, S. Y.; Zhao, S. N.; Hao, Z. M.; Zhu, M.; Meng, X.; Wu, L. L.; Zhang, H. J.: Single-crystal-to-single-crystal transformation of a europium(III) metal-organic framework producing a multi-responsive luminescent sensor. Adv. Funct. Mater. 24 (2014) 4034-4041. 
3. Bu, X. H.; Tong, M. L.; Chang, H. C.; Kitagawa, S.; Batten, S. R.: A neutral 3D copper coordination polymer showing 1D open channels and the first interpenetrating NbO-Type network. Angew. Chem., Int. Ed. 43 (2004) 192-195.

4. Murray, L. J.; Dinca, M.; Long, J. R.: Hydrogen storage in metal-organic frameworks. Chem. Soc. Rev. 38 (2009) 1294-1314.

5. Meng, X.; Song, S. Y.; Song, X. Z.; Zhu, M.; Zhao, S. N.; Wu, L. L.; Zhang, H. J.: A Eu/Tb-codoped coordination polymer luminescent thermometer. Inorg. Chem. Front. 1 (2014) 757-760.

6. Meng, X.; Zhong, R. L.; Song, X. Z.; Song, S. Y.; Hao, Z. M.; Zhu, M.; Zhao, S. N.; Zhang, H. J.: A stable, pillar-layer metalorganic framework containing uncoordinated carboxyl groups for separation of transition metal ions. Chem. Commun. 50 (2014) 6406-6408.
7. Allendorf, M. D.; Bauer, C.A.; Bhakta, R. K.; Houk, R. J. T.: Luminescent metalorganic frameworks. Chem. Soc. Rev. 38 (2009) 1330-1352.

8. Zhu, M.; Song, X. Z.; Song, S. Y.; Meng, X.; Zhao, S. N.; Wu, L. L.; Wang, C. ; Zhang, H. J.: A temperature-responsive smart europium metal-organic framework Sswitch for reversible capture and release of intrinsic $\mathrm{Eu}^{3+}$ ions. Adv. Sci. 2 (2015) 1500012.

9. Dechambenoit, P.; Long, J. R.: Microporous magnets. Chem. Soc. Rev. 40 (2011) 3249-3265.

10. Zhao, S. N.; Li, L. J.; Song, X. Z.; Zhu, M.; Hao, Z. M.; Meng, X.; Wu, L. L.; Feng, J.; Song, S. Y.; Wang, C.: Lanthanide Ion Codoped Emitters for Tailoring Emission Trajectory and Temperature Sensing. Adv. Funct. Mater. 25 (2015) 1463-1469.

11. Sheldrick, G. M.: A short history of SHELX. Acta Crystallogr. A64 (2008) 112-122. 